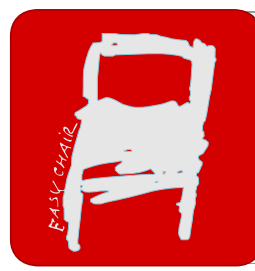

EPiC Series in Health Sciences

\title{
Registration of Knee Kinematics with a Navigation System. A Validation Study.
}

\author{
Florent Baldairon, Noah Davis, Lindsey Palm, Scott Banks, Jean-Yves \\ Jenny \\ University Hospital Strasbourg, Strasbourg, France \\ jean-yves . jenny@chru-strasbourg.fr
}

\begin{abstract}
The specific navigation system used can be currently considered as a reliable instrument for measuring the kinematics of a TKA for HKA angle and medial and lateral gaps, but non for femoral translation and rotation.
\end{abstract}

\section{Introduction}

Total knee arthroplasty (TKA) is considered a highly successful procedure. Survival rates of more than $90 \%$ after 10 years are generally reported (Baumbach 2016). The restoration of physiological kinematics is one of the goals of a TKA (Meneghini 2017). Navigation systems have been developed to allow an accurate and precise placement of the implants (Jenny 2005). But its application to the intraoperative measurement of knee kinematics has not been validated. The hypothesis of this study was that the measurement of the knee axis, femoral rotation, femoral translation with respect to the tibia, and medial and lateral femorotibial gaps during continuous passive knee flexion by the navigation system would be different from that by fluoroscopy taken as reference. 


\section{Material and methods}

Five pairs of knees of preserved specimens were used. The e.Motion FP ${ }^{\circledR}$ TKA (B-Braun Aesculap, Tuttlingen, Germany) was implanted using the OrthoPilot TKA 4.3 version and Kobe version navigation system (B-Braun Aesculap, Tuttlingen, Germany). Kinematic recording by the navigation system was performed simultaneously with fluoroscopic recording during a continuous passive flexion-extension movement of the prosthetic knee. Kinematic parameters were extracted from the fluoroscopic recordings by image processing using JointTrack Auto ${ }^{\circledR}$ software (University of Florida, Gainesville, USA) (Banks 1996). This software uses a matching algorithm based on the method described by Mahfouz (Mahfouz 2003): the 3D model of the implant and the image of the implant on the radiograph are rendered completely white on a black background. The border between the white and black region is then extracted from this image. Next, a "grow" operation is performed, which codes each pixel to a low distance from the contour with a score that is inversely proportional to its distance from the contour. This allows points close to the contour to contribute to the score. corresponding to a quantity proportional to their proximity. Edge detection results in a binary image with non-zero values along the contours, and zero values everywhere else. The shape correspondence between the two images is evaluated on the basis of a weighted combination of two measures. The first 67 measurement is a correlation on the intensity values of the two images, the second one is a measurement is a correlation on the contours of the shape. The already published results of this system indicate that knee rotations can be measured with an accuracy of approximately one degree and that sagittal plane translations can be measured with an accuracy of approximately $0.5 \mathrm{~mm}$ (Banks 1996).

The main criteria were the axis of the knee measured by the angle between the center of the femoral head, the center of the knee and the center of the ankle (HKA), femoral rotation, femoral translation with respect to the tibia, and medial and lateral femorotibial gaps. The data analysis was performed by a Kappa correlation test. The agreement of the measurements was assessed using the intraclass correlation coefficient (ICC) and its $95 \%$ confidence interval. ICC over 0.75 was arbitrarily considered as satisfactory. 


\section{Results}

The differences between navigated and fluoroscopic recordings are reported in figures 1 to 4 .

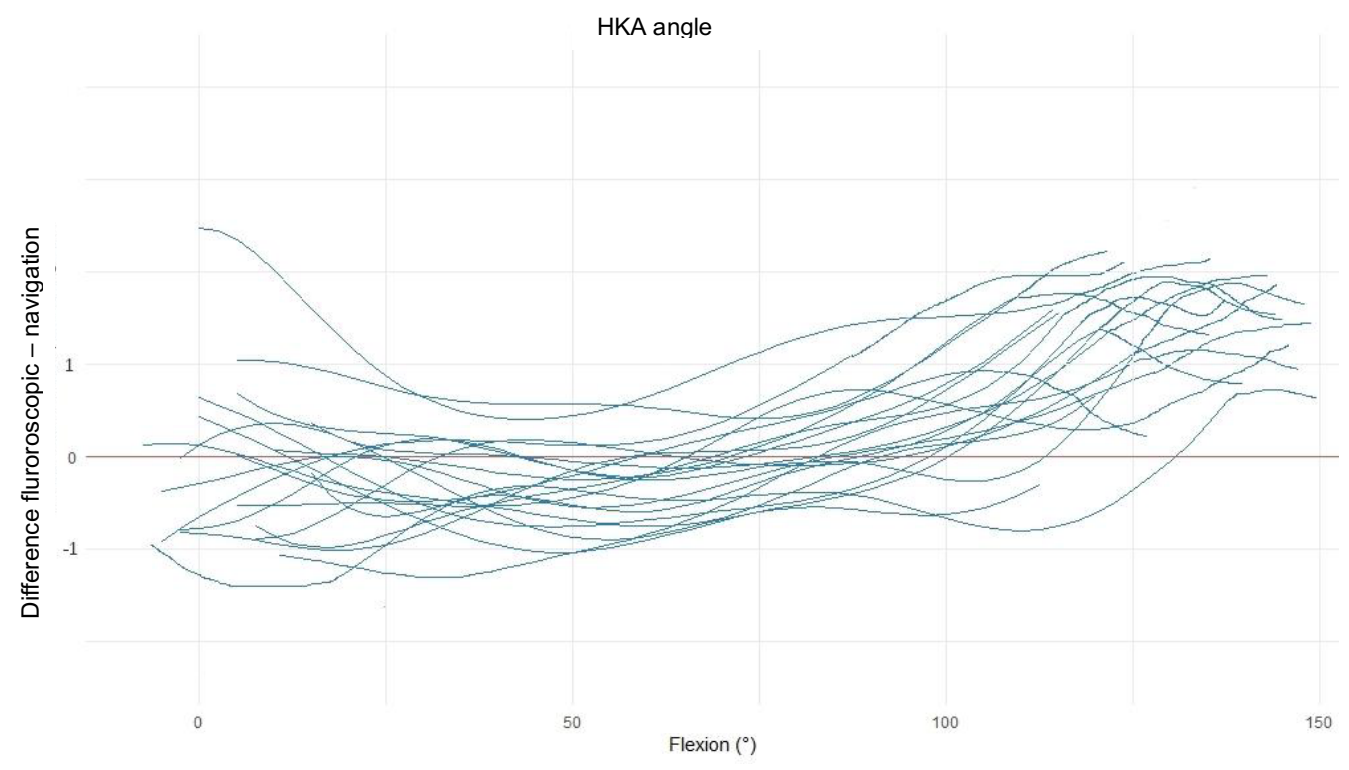

Figure 1: HKA angle 
Figure 2: Femoral translation

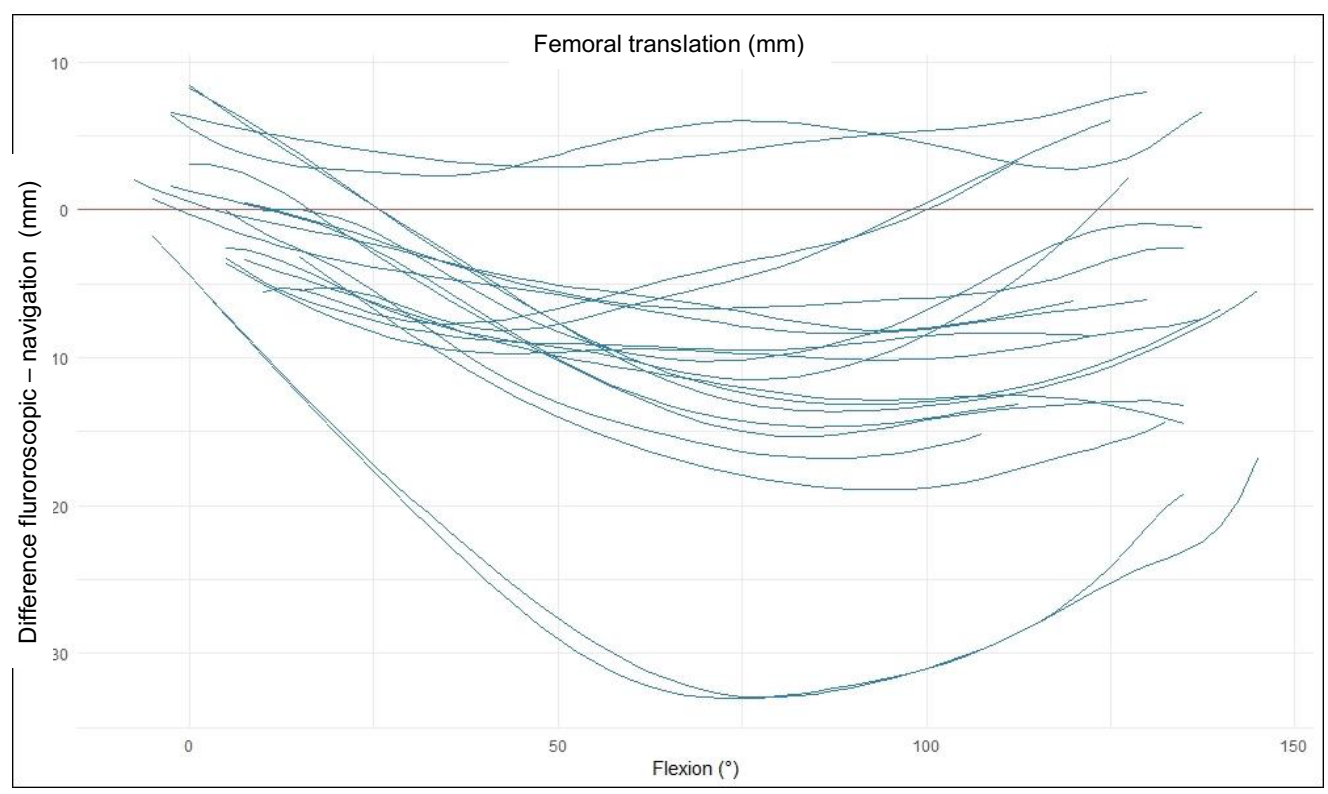

Figure 3: Femoral rotation

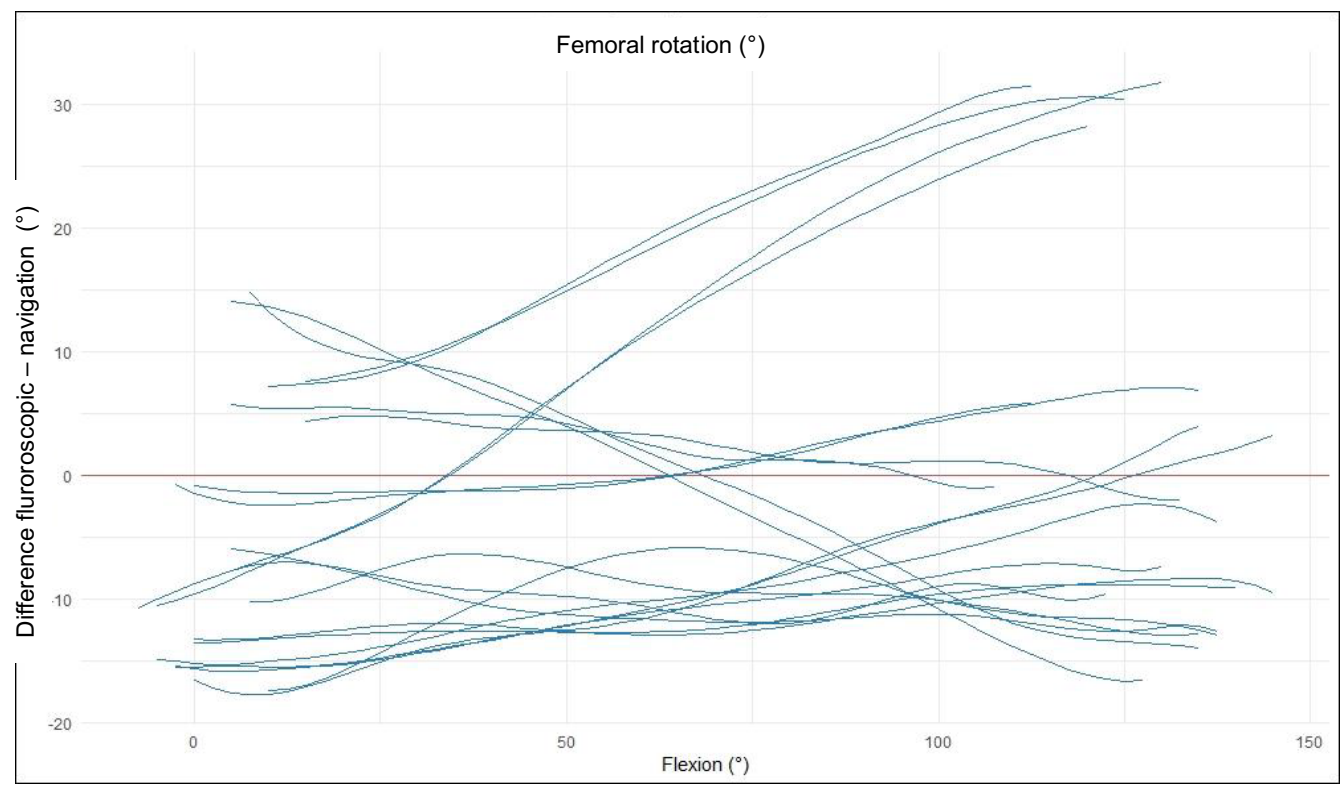


Figure 4: Medial gap

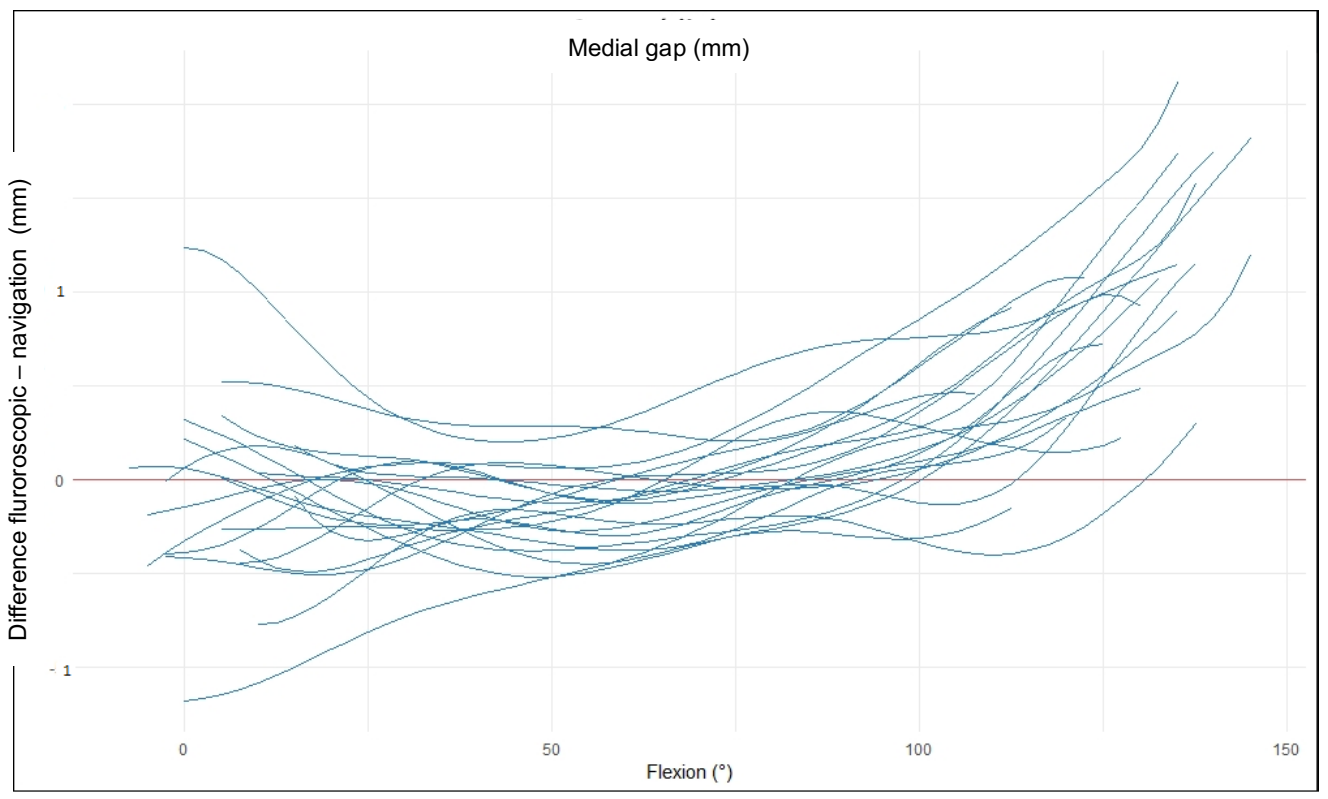

Figure 5: Lateral gap

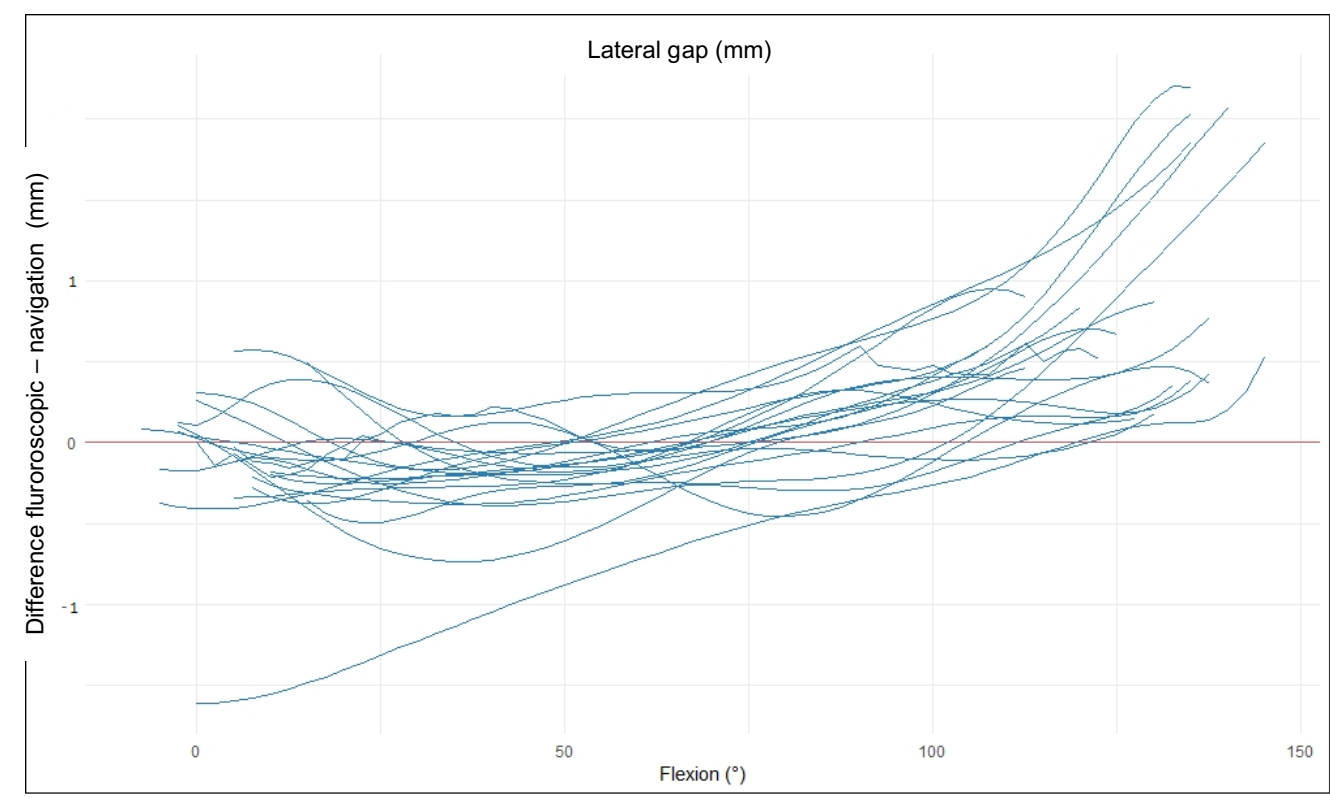

The respective CCIs were as follows: HKA angle 0.839 [0.820;0.856]; femoral translation 0.560 [0.517; 0.600]; femoral rotation 0.652 [0.616; 0.686]; medial femorotibial gap 0.905 [0.894; 0.916]; lateral femorotibial gap $0.767[0.740 ; 0.791]$. 


\section{Discussion}

Fluoroscopic analysis of the knee kinematics has been validated for postoperative analysis and is considered as the current gold standard (Kanekasu 2004, Prins 2014, Angerame 2019). It makes then sense to compare the intra-operative navigated analysis to this reference.

Correlation between measurements of HKA angle by the two methods was satisfactory. Measurements of TKA kinematics by the navigation system and by fluoroscopy were consistent for this measurement. The used navigation system may be considered as a reliable tool to assess the HKA angle and its variation during knee flexion.

Correlation between measurements of medial and lateral femorotibial gaps by the two methods was satisfactory as well. Measurements of TKA kinematics by the navigation system and by fluoroscopy were consistent for this measurement. The used navigation system may be considered as a reliable tool to assess the medial and lateral femorotibial gaps (i.e. medial and lateral femorotibial laxity) and their variation during knee flexion

Correlation between measurements for femoral translation and femoral rotation were poor. Measurements of TKA kinematics by the navigation system and by fluoroscopy were not consistent for these both measurements. These differences can be explained by a methodological bias: the femoral reference frames of both systems were different.

At the end of this work, the specific navigation system cannot be considered as a reliable instrument for measuring the complete kinematics of a TKA. It still measures reasonably HKA angle and medial and lateral femorotibial gaps. A simple mathematical calculation might compensate for the biases observed for measurements of femoral translation and femoral rotation.

\section{References}

1. Banks SA, Hodge WA, Accurate measurement of three-dimensional knee replacement kinematics using sdingle-plane fluoroscopy, IEEE Trans Biomed Eng, 43(6), pp: 638-649, 1996.

2. Baumbach JA, Willburger R, Haaker R, Dittrich M, Kohler S, 10-year survival of navigated versus conventional TKAs: a retrospective study, Orthopedics, 39(3 Suppl), pp: S72-76, 2016.

3. Meneghini RM, Deckard ER, Ishmael MK, Ziemba-Davis M, A dual-pivot pattern simulating native knee kinematics optimizes functional outcomes after total knee arthroplasty. $\mathrm{J}$ Arthroplasty, 32(10), pp: 3009-3015, 2017.

4. Jenny JY, Clemens U, Kohler S, Kiefer H, Konermann W, Miehlke RK, Consistency of implantation of a total knee arthroplasty with a non-image-based navigation system: a case-control study of 235 cases compared with 235 conventionally implanted prostheses, J Arthroplasty, 20(7), pp: 832-839, 2005.

5. Mahfouz MR, Hoff WA, Komistek RD, Dennis DA, A robust method for registration of three-dimensional knee implant models to two-dimensional fluoroscopy images, IEEE Trans Med Imaging, 22(12), pp:1561-1574, 2003. 
6. Prins AH, Kaptein BL, Banks SA, Stoel BC, Nelissen RG, Valstar ER, Detecting condylar contact loss using single-plane fluoroscopy: a comparison with in vivo force data and in vitro biplane data, J Biomech, 7;47(7), pp: 1682-1688, 2014.

7. Kanekasu K, Banks SA, Honjo S, Nakata O, Kato H, Fluoroscopic analysis of knee arthroplasty kinematics during deep flexion kneeling, J Arthroplasty 19(8), pp: 998-1003, 2004.

8. Angerame MR, Holst DC, Jennings JM, Komistek RD, Dennis DA, Total knee arthroplasty kinematics, J Arthroplasty 34(10), pp: 2502-2510, 2019.

\section{Disclosure}

Florent BALDAIRON has nothing to disclose. Noah DAVIS has nothing to disclose. Lindsey PALM has nothing to disclose. Scott BANKS has nothing to disclose. Jean-Yves JENNY receives royalties from AESCULAP, is a paid consultant for FH Orthopedics and Globus Medical, is member of the board of the CAOS-International Society and of the International Society for Technology in Arthroplasty. 